\title{
Non Cystic Fibrosis Bronchiectasis-new clinical approach, management of treatment and pulmonary rehabilitation
}

\author{
Anca Maierean ${ }^{1}$, Teodora Gabriela Alexescu ${ }^{2}$, Lorena Ciumarnean ${ }^{2}$, Nicoleta Motoc ${ }^{1}$, Ana Chis ${ }^{1}$, \\ Maria Victoria Ruta ${ }^{3}$, Gabriela Dogaru ${ }^{4}$, Maria Aluas ${ }^{5}$
}

Corresponding author: Anca Maierean, E-mail: anca.lupascu91@gmail.com,

(cc) BY-NC-ND Balneo Research Journal DOI: http://dx.doi.org/10.12680/balneo.2019.247 $\quad$ Vol.10, No.2, May 2019 p: 103-113

1. 'Iuliu Hatieganu’University of Medicine and Pharmacy, Department of Pneumology, Cluj - Napoca, Romania 2. „Iuliu Hatieganu” University of Medicine and Pharmacy, Department of Internal Medicine, Cluj - Napoca, Romania

3. „Iuliu Hatieganu”, University of Medicine and Pharmacy, Department of Physiology, Cluj - Napoca, Romania 4. „Iuliu Hatieganu”, University of Medicine and Pharmacy, Department of Medical Rehabilitation, Clinical Rehabilitation Hospital, Cluj-Napoca, Romania

5. „Iuliu Hatieganu”, University of Medicine and Pharmacy, Department of Medical Education, Cluj - Napoca, Romania Abstract

Non-Cystic Fibrosis Bronchiectasis (NCFB) are characterised by abnormal, permanently damaged and dilated bronchi due to the innapropiate clearence of various microorganisms and recurrent chronic infections. The diagnosis is suggested by the clinical presentation and is confirmed by multiple investigations. There are some comorbidities associated with bronhciectasis, such as chronic obstructive pulmonary disease (COPD), cardiovascular disorders, gastro-esophageal reflux disease (GERD), psychological illnesses, pulmonary hypertension, obstructive apnea syndrome(OSA). The condition has a substantial socioeconomic impact because it requests a multidisciplinary management and periods of exacerbations are common. The aims of the management of bronchiectasis are to reduce symptoms (such as sputum volume and purulence, cough and dyspnea), reduce the frequency and severity of exacerbations, preserve lung function and improve health-related quality of life. The multidisciplinary approach of bronchiectasis patients require along with the medical treatment, a specific plan of nonphamarcological strategies, including balneological intervention. There are a lot of techniques improving the airway clearence, such as: active cycle of breathing techniques (which include breathing control, thoracic expansion exercises, forced expiratory technique), oscilatting possitive expiratory pressure, autogenic drainage, gravity-assisted-positioning, modified postural drainage. Together with specific medication, these techniques can diminuate symptoms and improve the quality of life.

Key words: $N C F B$, airway clearence, physiotherapy,

\section{Introduction}

Non-Cystic Fibrosis Bronchiectasis (NCFB) is a chronic debilitating condition, first described by René Laennec in 1819. This condition is characterised by abnormal, permanently damaged and dilated bronchi, due to chronic bronchial inflammation because of inappropriate clearance of various microorganisms and recurrent or chronic infection (1). However, in a substantial number of patients no cause is found (2). Symptoms vary from intermittent episodes of respiratory infections with excessive mucus production to chronic symptomes with persistent daily expectoration of purulent sputum. It is a debilitating illness responsible for significant morbidity, with patients typically suffering from intermittent episodes of respiratory infections with excessive mucus production to chronic symptoms such as a persistent cough, daily sputum expectoration, recurrent chest infections and a poor health-related quality of life (3). The condition has a substantial socioeconomic impact because it request multidisciplinary management and periods of exacerbations are common (4).

\section{Prevalence}

Even if once NCFB was a very rare diagnosis, in the last two decades its prevalence has varied among populations and several studies have reported a prevalence ranging from 486 to 1106 per 100000 persons (5-7). The incidence of non-cystic fibrosis bronchiectasis is $2-5$ patients per 1,000 population (8). It was observed that this pathology is more frequent in women and older individuals and many patients tend to have a more severe and symptomatic disease. Bellelli $\mathrm{G}$ et al. reported in one study of over 1,200 patients with bronchiectasis, $50 \%$ were over 65 years old and $19.1 \%$ were over 75 years (9). Increasing age is recognized as an independent risk factor for bronchiectasis severity (10). There is still a high incidence in developed countries and globally with the increased life expectancy of the general population, there is a greater risk of more people developing chronic illness, including bronchiectasis (11). 


\section{Patophysiology}

The aetiology of NCFB is variable, but the common pathophysiological pathway represents a vicious cycle containing three important elements: inflammation, infection and damage on the bronchial wall. Potential causes of this breach include severe lower respiratory tract infections, gastric aspiration and/or inhalation of toxic gases, immunglobulin deficiency, ciliary disfunction, connective tissue disease, rheumatoid arthritis-related, airways disease (asthma/COPD), non-tuberculosis mycobacterium, foreign body inhalation, inflammatory bowel diseaserelate. These modifications, through local or systemic inflammation, cause disturbances in the architecture of bronchial airways $(12,13)$.

The usual mucociliary clearance defence mechanism is impaired, so the airways become chronically infected with a variety of bacteria which promotes further neutrophilic inflammation. Once the bacterial density in the airways is increasing, there is an exaggerated inflammatory response consisting of greater numbers of neutrophils and higher concentrations of neutrophil degradation products beside other inflammatory markers (14).

So, the patient with NCFB is enrolled in a vicious cycle of infection and inflammation developing persistent symptoms, frequent exacerbations and further airway damage (15). These modifications such as host inflammatory response and microbial cytotoxins, cause additional structural damage of the lung and further impair mucus clearence, so it is easier for the microorganisms to form biofilms (16). The role of the biofolms is to protect the bacteria from the clearence of the host immune system which reduce the effects of antibiotics further potentiating airway inflammation (17).

\section{Clinical Presentation}

Non-cystic fibrosis bronchiectasis can be presented as asymptomatic, or in some individuals can cause massive haemoptysis and respiratory failure, so the most common clinical presentation is between these extremes. In one study of 103 adults with NCFB, 98\% reported a chronic cough, and $76 \%$ of patients had daily sputum production. From common presenting symptoms, dyspnoea occured in $62 \%$ of cases and chronic fatigue was reported by $74 \%$ of subjects $(18,19)$. Moreover, intermittent haemoptysis occurred in $26-51 \%$ of cases, which at times became life-threatening (19). On the other hand, many patients with bronchiectasis require frequent hospital admissions for recurrent pneumonia(18) and depression or anxiety which may significantly affect their quality of life (20).

\section{Investigations}

Physical examination and history contribute to the diagnosis of bronchiectasis. The most common modifications include crackles ( $73 \%$ of subjects), rhonchi (44\%) and wheezing (21\%-34\%) (18).

The first tool to evaluate a patient with bronchiectasis is a standard chest radiography which can detect multiple areas of bronchial wall dilatation in subject with moderate to severe bronchiectasis. However, studies have evidenced that chest radiography has moderate sensitivity $(88 \%)$ and poor specificity (74\%) for the detection of bronchiectasis (21).

The current gold standard for the diagnosis and confirmation of bronchiectasis is high-resolution computed tomography $(\mathrm{CT})$. The characteristic feature of NCFB by CT is bronchial wall dilatation with the luminal diameter greater than 1-1.5 the size of the accompanying pulmonary artery branch. Other findings include absence of normal tapering of the bronchi, bronchial wall thickening, mucoid plugging of the airways and proximity of visible airways close to the pleura (22).

On the other hand, pulmonary function testing is often used to evaluate the subject with chronic pulmonary diseases. Patients with bronchiectasis usually present mild to moderate airway obstruction, with a forced expiratory volume in the 1st second (FEV1)/forced vital capacity (FVC) ratio less than 70 and a FEV $1>50 \%$ of predicted. In $22 \%$ of cases with NCFB, there is a significant bronchodilatator response(18). Some conditions are associated with more severe obstruction, such as: a history of Pseudomonas infection or colonisation, multilobar involvement of disease, greater purulent sputum volume and those patients with at least four exacerbations over a 2-year period (23).

In addition, sputum culture must be obtained from all patients with bronchiectasis. The most common organisms initially isolated are Gram negative bacteria including Haemophillus infuenzae (47\%), Pseudomonas aeruginosa $(12 \%)$ and Moraxella catarrhalis $(8 \%)$. After a period of time, Pseudomonas aeruginosa becomes more frequent. All in all, Grampositive bacteria such as Streptococcus pneumoniae and Staphylococcus sureus are rare (24). 
In the last decades, Non-tuberculosis mycobacterium (especially Mycobacterium avium) incidence has increased accounting for $2-30 \%$ of pathogens isolated (25).

Once bronchiectasis is confirmed based on clinical presentation and radiographic imaging, determining the underlying cause is important to effective therapeutic management. First of all, both primary and secondary immunoglobulin deficiency (in particular IgG deficiency) should be tested because it can be associated with bronchiectasis and the frequency of infections can often be reduced by immunoglobulin replacement therapy. All patients should undergo testing for allergic bronchopulmonary aspergillosis(ABPA), depending on radiological features (temporary infiltrates are seen initially and it can eventually lead to proximal bronchiectasis) and tests to confirm aspergillus hypersensitivity (raised peripheral blood eosinophils, total IgE, Aspergillus specific IgE or positive skin test with or without aspergillus precipitins) (26).

\section{Comorbidities}

The possibility of coexisting inflammatory bowel disease, connective tissue disease or vasculitis. Comorbidities are more likely in the elderly and clinical suspicion will warrant relevant screening such as ANCA, anti-CCP, fecal calprotectin etc. Bronchiectasis associated with a connective disease such as rheumatoid arthritis is often more aggressive and carries a worse prognosis (26).

In addition, several other pathologies may occur at any stage of bronchiectasis and are likely major contributors to increased hospitalisations, healthcare utilisation and socioeconomic costs (27). These include COPD, cardiovascular disorders, gastroesophageal reflux disease (GERD), psychological illnesses, pulmonary hypertension, obstructive apnea syndrome(OSA), cognitive impairment, lung, oesophageal and hematological malignancies (20,2829). One of the important comorbidities is OSA, but the relationship between sleep disorders and bronchiectasis has not been well described. There is a assumption that, due to the irreversible dilatation of the bronchi, the presence of secretions, and airflow obstruction, patients with non-cystic fibrosis bronchiectasis may be predisposed to hypoxemia during sleep, or to symptoms that may lead to arousal (30).
The importance of screening programs for comorbidities, such as OSA and diabetes mellitus type 2 is evident in bronchiectasis patients, moreover because some studies showed that only being overweight itself produces a greater risk of developing a metabolic syndrome and sleep disorders (31).

\section{Management}

The aims of the management of bronchiectasis are to reduce symptoms (such as sputum volume and purulence, cough and dyspnea), reduce the frequency and severity of exacerbations, preserve lung function and improve health-related quality of life.

In the multidisciplinary management, every respiratory phisician must take into account the gravity score. There are several scales of severity. One of them is the one proposed by Chalmers et al. who introduced the bronchiectasis severity index (BSI), including High resolution Computed Tomography (HRCT) score, FEV1, Medical Research Council dyspnoea score, bacterial colonisation (Pseudomonas aeruginosa or other pathogenic bacteria), prior hospital admission and exacerbations. They conclude that the BSI was a sensitive tool in predicting future risks of hospitalisation(10). On the other hand, MartinexGarcia et al. (32), established the FACED score, a simpler one, which includes FEV1, age, Pseudomonas aeruginosa colonisation, radiological extension and dyspnoea. Similarly, the FACED score effectively predicted mortality.

The first step of the management is the patients' education; they should understand the diagnosis in order to limit other injury to the airways, by smoking cessation, limiting infective insults, prevention of viral infections by receiving the annual seasonal influenza vaccination (33-34). Also, in obese patients, diet is one of the most important interventions along with a training program in order to reduce body mass index (BMI), improve sleep quality and increase respiratory muscle endurance in patients with bronchiectasis and other comorbities, such as OSA, but in selected cases with associated craniofacial deformities, early orthodontic treatment could be necessary $(35,36,37)$. Also, weight loss improves the cardiovascular status, moreover because obese patients develop a metabolic syndrome which can be fatal for bronchiectasis patients because it can reduce their effort capacity and their quality of life (38). 
The multidisciplinary approach of bronchiectasis patients requires along with the medical treatment, a specific plan of nonphamarcological strategies, including balneological intervention: an active cycle of breathing techniques (which include breathing control, thoracic expansion exercises, forced expiratory technique), oscilatting positive expiratory pressure, autogenic drainage, gravity-assistedpositioning, modified postural drainage.

\section{Airway clearance}

Airway clearance techniques (ACT) are nonpharmacological strategies used worldwide in order to improve symptoms, reduce exacerbation frequency and increase quality of life (39). There are some short terms goals of ACT such as more effective sputum clearance which has been proven to improve ventilation, reduce cough impact and breathlessness. On the other hand, its long-term effects are reducing airway damage by stopping the vicious cycle of colonization and subsequent inflammation, reducing the number of exacerbations and hospitalizations and improving the quality of life (40).

ACT are a key component in the management of patients with bronchiectasis, moreover for patients with a chronic productive cough or difficulty expectorating sputum which may benefit from regular twice daily ACT as recommended in the current guidelines (41). Active Cycle of Breathing Technique (ACBT) is a repetitive cycle consisting of 3 distinct components: breathing control (BC), thoracic expansion exercises (TEE), and the forced expiration technique (FET). The cycle and length of each component are flexible and can be adapted to individual needs. $\mathrm{BC}$ is relaxed tidal breathing using the lower chest and performed at the patient's own rate. Its role is to minimize any flow obstruction. TEE are dynamic procedures and are performed by taking deep slow inspirations through the nose (if possible), followed by an inspiratory pause with an open glottis, and subsequently relaxed expiration, in order to mobilize the secretions. The FET is a principle component of the ACBT and is defined as a combination of 1 or 2 forced expirations (huffs) interspersed with periods of BC. The lung volume can be varied depending on which segment to target. For example, FET from high lung volumes helps to clear secretions, whereas FET from low lung volumes helps to mobilize secretions. The FET aims to move the mobilized secretions to the proximal airways to be cleared, which then may subsequently require a cough (42). However, in practice ACT remains underutilized, as showed by The European Bronchiectasis Data Registry (EMBAC) that reported that only $45 \%$ of patients perform ACT regularly $(43,44)$. ACT has two main principles: to ventilate the regions distally, behind the obstruction and to modulate the expiratory airflow to propel secretions proximally up the airways. So, to enhance the mucus clearance, the peak expiratory flow rate should be at least 30-60 1/min and greater than the inspiratory flow (44). Other techniques used in patients with bronchiectasis are: oscilatting positive expiratory pressure (PEP), autogenic drainage (AD) and ELTGOL (active technique where the patients carries out slow expiration with the glottis open in a lateral decubitus position).

PEP uses a set resistance that is applied throughout expiration to enhance the mobilization of secretions, by using either a mask or a mouthpiece attachment and induces a temporary increase in functional residual capacity. Moreover, it facilitates collateral ventilation, and encourages the recruitment of closed airways. Furthermore, it may splint airways open, aiding expiratory airflow, because airways are often prone to collapse during active expiration as a result of increased intrathoracic pressure $(45,46)$. Autogenic drainage $(\mathrm{AD})$ is a 3-phase breathing programme using high expiratory flow rates at varying lung volumes to facilitate mucus clearance. It requires a progressive volume increase in order to maximize expiratory flow velocity to produce shearing forces and move secretions. Inspiration should be low flow with an inspiratory pause and open glottis. Expiration should be a higher flow, although aiming to minimize airway collapse $(47,48)$.

Considering the studies, Patterson et al. demonstrated that ACBT versus oscilatting PEP had no improvement in lung function or sputum weight over a 10-14 day treatment period in 20 stable patients (49). This idea was sustained in the study of Thompson et al. who also took into consideration the quality of life, which was not improved after a 4-week treatment period (50). In a study performed by Herrero Cortina et al., both $\mathrm{AD}$ and ELTGOL resulted in significantly greater sputum compared to the control group (51). Anther study, involving ELTGOL, made by Munoz et al. which compared the ELTGOL technique to placebo exercises twice daily in 44 patients over a 1-year period, reported fewer 
exacerbations, reduced cough impact and improvement in the quality of life in the therapy group (52).

Another technique is gravity-assisted positioning (GAP) which is passive and relies solely on the effects of gravity and changes in regional ventilation with positioning, so it cannot be used alone as an ACT. Patient position themselves to drain specific lobes within the lungs, using gravity to enhance movement of secretions. For example, the lower and middle zones of the lung drain includes a head-down tip position. Studies showed that the performance of ACTs in GAP positions may help enhance sputum clearance (53). Modified postural drainage (MPD) uses similar specific lobar drainage positions as in GAP, but eliminates any head-down tip, thus using gravity neutral positions. MPD is recommended in patients that have contraindications for GAP, for examples in subjects which are associated with GORD. Cecins et al assessed the effectiveness of modified positions without a head-down tip and showed no significant difference in MPD versus GAP, because the results of sputum weight did not vary more than $15 \%$ (54).

These studies with interventions longer than a single treatment demonstrate some proof of the concept on the effectiveness of ACT in bronchiectasis and support the general consensus that currently no one ACT is superior in terms of clinical outcomes (55). There is wide variety of techniques that are age, preference and co-morbidity dependent, so the selection of ACT should be targeted according to the patient's individual characteristics (56). Taking into consideration the comorbidities, such as OSA, the airway clearance is very important because the presence of various microbiological and inorganic structures on the inner surface of CPAP masks and tubes emphases the risk of microbial and inorganic elements inhalation into the upper and lower airways. Therefore, the microclimate and the hygiene of CPAP device, including masks and tubing is very important in order to reduce exacerbations (57-58). Also, patients with bronchiectasis should reduce their exacerbation risk by limiting air pollution both indoor and outdoor and the exposure to allergenics (59).

\section{Airway humidification}

The aim of airway humidification (with molecular water added to gas) is to reduce mucus viscosity and enhance flow through the airways. There are a few studies on this subject, which demonstrated that airway humidification increases airway clearance and sputum yield, so can be used in addition to ACT. However, the evidence is insufficient to recommend it for routine use in subjects with bronchiectasis (60).

\section{Mucoactive therapy}

Studies showed that mucoactive therapy should be considered if ACT are not effective (41). Mucoactive medications potentially increase the ability to expectorate sputum and/or decrease mucous hypersecretion and should be coordinated with ACT to ensure the overall effect is optimized. As Balsamo et al. showed, they can broadly be characterized into several major groups based on their potential mechanism of action (61):

1. Expectorants are drugs that induce discharge or expulsion of mucous from the respiratory tract. Typically, this requires a coughing action to loosen and bring up the mucous from the lungs or upper respiratory tract. Examples include hypertonic saline (HTS) and a solution of manitol.

2. Mucoregulators: drugs that regulate mucous secretion or interfere with the DNA/F-actin network. Examples include carbocisteine and anticholinergic agents.

3. Mucolytics: drugs that decrease mucous viscosity. Examples include N-acetylcysteine, erdosteine and DNase.

4. Mucokinetics: drugs that increase mucociliary clearance by acting on the cilia. Also referred to as cough clearance promoters. Examples include bronchodilators and surfactants (61).

The European Respiratory Society (ERS) guidelines (62) summarize the findings of three systematic reviews that have comprehensively examined the current evidence for mucoactives (63-65). It was proven that none of the mucoactive agents significantly reduced the number of exacerbations, but one trial with 88 participants showed that high doses of bromhexine with antibiotics significantly eased difficulty in expectoration and sputum production days compared to a placebo (66). HTS action is achieved by altering the concentration of water in the mucus but, clinical trials are limited. However, it was evidenced that it is responsible for yielding greater sputum weights with greater ease and less viscosity (67). The manitol role is to enhacemucocilliary clearance with improved mucus 
clearance for up to 24 hours after inhalation and it was demonstrated that it has an important positive effect on small airways function, cough tansportability and health-related quality of life (68). The prescription of mucoactive therapies should be done in addition to ACT and therefore it is important that the mechanism of action of mucoactive drugs and their timing with ACT are taken into consideration. In case of HTS which has a short-term/immediate effect, the ACT should be done immediately after HTS inhalation (69). There are two active clinical trials exploring the efficacy of commonly used mucoactives (HTS and carbocisteine) in bronchiectasis. The results are likely to have an important impact on future practice (7071), moreover because there is stronger evidence for carbocisteine and HTS in other conditions and these are the most commonly usedmucoactives in bronchiectasis (72). Regarding inhaled mucolytics, their role is to cleave the excessive quantities of DNA accumulated due to neutrophil activity, reduce sputum viscosity, improve expectoration, airway clearance and lung function. However, it is not recommended in routine use because of its side effects with the decline of FEV1, higher rate of exacerbations, increased use of antibiotics and a greater rate of hospitalisations (73). On the other hand, inhaled corticosteroids are recommended to be used in the treatment of bronchiectasis together with rehabilitation programmes where there is co-existing obstructive airways disease such as asthma, COPD or ABPA (74).The British Thoracic Society (BTS) bronchiectasis guidelines recommend a trial of bronchodilator therapy if shortness of breath affects the patient's activities of daily living. In these situations it is worthwhile to perform a trial of shortacting beta-agonist (SABA) or short-acting muscarinic-antagonist (SAMA) initially and if there is clinical benefit then proceed to a long-acting bronchodilator (75).

\section{Antimicrobial therapy}

The number of clinical trials investigating antibiotic therapy in NCFB is small, but has been administrated systemically or via inhalation (76). However, chronic treatment is not recommended and the most effective antibiotics are macrolides. They act with an anti inflammatory effect, reducing the total bronchoalveolar lavage cell count, sputum volume and interleukine 8 and neutrophil activity. Also, Anwar et al. showed an improvement in exacerbation frequency, spirometry and sputum microbiology when treated with azithromycin $250 \mathrm{mg}$ three times a week (77). However, inhaled antibiotics have more limited side effects, but may be less tolerated because of the bronchospasm. For example, inhaled tobramycin decreased the number of admissions and days in the hospital and it has been showed that by prolonging their use may appear a positive effect. Also, there are continuous improvements in drug design, with aerolised formulations that are well tolerated by the epithelial surface, able to penetrate infected sputum and capable of avoiding immune system clearance in order to achieve a better therapeutic response (78).

\section{Pulmonary rehabilitation}

Pulmonary rehabilitation, with patient-centred exercise training and collaborative self-management education delivered by an interdisciplinary team and centred on the specific needs of the patient (30), has evolved beyond the confines of chronic obstructive pulmonary disease (COPD) management. Pulmonary rehabilitation must take into account the patients' comorbidities. In general, PR incorporates several exercise modalities (treadmill walking, cycle ergometry, weight lifting and arm ergometry) and patient education and works through secondary impairments such as peripheral muscle dysfunction, psychosocial dysfunction and maladaptive behaviours (79). It is necessary that patients be educated once the pulmonary rehabilitation programme is initiated to minimize their exposures to respiratory irritants. In this direction, smoking cessation is crucial in addition to the avoidance of air pollution, boot outdoor and indoor (radon exposure) (80,81,82). Exercise training (ET) programmes are comprised of the exercise modalities without an educational component. PR and ET programmes are generally aimed at improving both upper limb and lower limb endurance and are tailored to the needs of the individual patient. The location of the programmes vary: some are incorporated into hospital-based physical medicine and rehabilitation medicine facilities; others are free standing and may even be in a community centre or in an outpatient physiotherapy department. Based on the location of the exercises, the trained stuff may include: exercise physiologists, respiratory care practitioners, physiotherapists and nurses. Also, the patients participating in PR or ET are generally monitored 
with oximetry and occasionally cardiac monitoring. The duration of the programs vary: in the United States, these programmes are generally comprised of an initial intensive phase which includes 36 1-hour sessions over 12 weeks followed by a selfguided maintenance phase, but in other parts of the world, the patients may receive shorter or longer periods of supervision (79).

A recent systematic review confirmed the short-term benefits that patients achieve from participating in supervised PR and ET, but noted that sustaining benefit is challenging (83). However, the study of Ong et al., suggested that despite the absence of a structured maintenance program, the 6-minute walking distance remained significantly higher than baseline at 12 months and the chronic respiratory disease questionnaire (CRQ) scores similarly remained stable for 12 months (84). In addition, Newall et al. which conducted the first study to assess the effects of exercise training in patients with bronchiectasis showed that an 8-week PR program was effective in improving the exercise capacity and health status, regardless of the addition of inspiratory muscle training (85). Moreover, Mandall et al., in one study comparing PR and chest physiotherapy versus chest physiotherapy alone in 30 patients with bronchiectasis, observed short-term improvement in PR outcomes, with a reduction in the frequency of exacerbation after 12 months, as well as an improvement in the walking distance (86).

The physiological rationale for $\mathrm{PR}$ and $\mathrm{ET}$ in bronchiectasis is that muscle weakness and physical inactivity may play a role in disease progression as well as impact the quality of life, frequency of infectious exacerbations and the ability to mobilize secretions (87). One observational study of 41 patients with bronchiectasis showed that PR significantly improved forced vital capacity and residual volume measurements (88). Lee et al, concluded in one study that compared ET with airway clearance training to standard care that there was an improvement in exercise capacity, dyspnoea and fatigue as well as fewer exacerbations over 12 months (89). Patients that cannot perform ET, can benefit from neuromuscular electrostimulation (NMES) which has been extensively used as a technique to improve muscle function and structure in different areas of rehabilitation and sport training programs (90).

\section{Conclusion}

Non cystic fibrosis bronchiectasis is a chronic, inflammatory lung disease that is characterized by a chronic cough, daily sputum production, and frequent exacerbations. There is a spectrum of disease, ranging from minor symptoms to a severe and progressive condition that can result in overwhelming infection or respiratory failure. It has become increasingly recognised that treatments for bronchiectasis cannot be extrapolated from other chronic respiratory diseases and more studies are required both to better understand the disease pathogenesis and to establish the optimum multidisciplinar approach to the management of this debilitating disease. Pulmonary rehabilitation is a comprehensive and effective treatment approach that improves overall clinical status and increases the level of physical activity in patients with bronchiectasis. It is essential that patients with bronchiectasis are included in comprehensive PR programs that are planned based on the patients' current clinical status and individual needs, in order to increase their quality of life.

\section{Conflict of interest}

There is no conflict of interest for any of the authors regarding this paper.

\section{References}

1. Stafler P, Carr SB, GoeminnW P, Dupont L. Non-cystic fibrosis bronchiectasis: diagnosis and management in 21st century. Postgrad Med J. 2010;86:493-501.

2. GoeminneP, Dupont L. Non-cystic fibrosis bronchiectasis: diagnosis and management in 21st century. Postgrad Med J. 2010;86:493-501.

3. Altenburg J, Wortel $\mathrm{K}$, van der Werf TS, Boersma WG. Non-cystic fibrosis bronchiectasis: clinical presentation, diagnosis and treatment, illustrated by data from a Dutch Teaching Hospital. Neth J Med. 2015;73:147-154.

4. Kelly Mg, Murphy S, Elborn JS. Bronchiectasis in secondary care: a comprehensive profile of a neglected disease. Eur J Intern Med. 2003;14:488-92.

5. Weycker D, Edelsberg J, Oster G, Tino G. Prevalence and economic burden of bronchiectasis. Clin Pulm Med. 2005;12:205209. 
6. Quint JK, Millett ERC, Joshi M, et al. Changes in the incidence, prevalence and mortality of bronchiectasis in the UK from 2004 to 2013: a population-based cohort study. Eur Respir J.2016;47:186-193.

7. Seitz AE, Olivier KN, Adjemian J, et al. Trends in bronchiectasis among Medicare beneficiaries in the United States, 2000 to 2007. Chest. 2012;142:432-439.

8. Quint JK, Millett ER, Joshi M, et al. Changes in the incidence, prevalence and mortality of bronchiectasis in the UK from 2004 to 2013: a population-based cohort study. Eur Respir J. 2016;47(1):186-193.

9. Bellelli G, Chalmers JD, Sotgiu G, et al. Characterization of bronchiectasis in the elderly. Respir Med. 2016;119:13-19.

10. Chalmers JD, Goeminne P, Aliberti S, et al. The bronchiectasis severity index. An international derivation and validation study. Am J Respir Crit Care Med. 2014;189(5):576-585.

11. Shoemark A, Ozerovitch L, Wilson R. Aetiology in adult patients with bronchiectasis. Respir Med .2007; 101:1163-70. doi:10.1016/j. Rmed.2006.11.008.

12. Pasteur MC, Bilton D, Hill AT; on behalf of the British Thoracic Society Bronchiectasis(non-CF) Guideline Group. British Thoracic Society guideline for non- CF bronchiectasis. Thorax. 2010; 65 (Suppl. 1): i1-i58.

13. Lonni S, Chalmers JD, Goeminne PC, et al. Etiology of non-cystic fibrosis bronchiectasis in adults and its correlation to disease severity. Ann Am Thorac Soc. 2015;12(12):1764-1770.

14. Fuschillo S, De FA, Balzano G. Mucosal inflammation in idiopathic bronchiectasis: cellular and molecular mechanisms. Eur Respir J. 2008;31:396e406.

15. Cole P. Inflammation: a two-edged sword-the model of bronchiectasis. Eur J Respir Dis Suppl. 1986;147:6-15.

16. Cole $\mathrm{P}$. The damaging role of bacteria in chronic lung infection.J Antimicrob Chemother. 1997;40(Suppl.A):5-10.

17. Chalmers JD, Hill AT. Mechanisms of immune dysfunction and bacterial persistence in non-cystic fibrosis bronchiectasis. Mol Immunol. 2013;55:27-34.

18. King PT, Holdsworth SR, Freezer NJ, et al. Characterisation of the onset and presenting clinical features of adult bronchiectasis. Respir Med. 2006;100:2183-2189.

19. Qi Q, Wang W, Li T, et al. Aetiology and clinical characteristics of patients with bronchiectasis in a Chinese Han population: a prospective study. Respirology. 2015;20:917-924.

20. Olveira C,Olveira G, Gaspar I, et al. Depression and anxiety symptoms in bronchiectasis :associations with health - related quality of life. Qual Life Res. 2013;22:597-605.

21. van der Bruggen-Bogaarts BA, van der Bruggen HM, van Waes PF, Lammers JW. Screening for bronchiectasis. A comparative study between chest radiography and highresolution CT. Chest. 1996;109: 608-611.

22. Bonavita J, Naidich DP. Imaging of bronchiectasis. Clin Chest Med. 2012;33:233248.

23. Guan WJ, Gao YH, Xu G, et al. Characterization of lung function impairment in adults with bronchiectasis. PLoS ONE. 2014;9:e113373.

24. King PT, Holdsworth SR, Freezer NJ, et al. Microbiologic follow- up study in adult bronchiectasis. Respir Med. 2007;101:16331638.

25. Maiz L, Giron R, Olveira C, Vendrell $M$, Nieto R, Martinez-Garcia MA. Prevalence and factors associated with nontuberculous mycobacteria in non-cystic fibrosis bronchiectasis: a multicenter observational study. BMC Infect Dis.2016;16:437.

26. Quinn TM, Hill AT. Non-cystic fibrosis bronchiectasis in the elderly: current perspective. Clinical Interventions in Aging .2018;13:16491656.

27. Joish VN, Spilsbury-Cantalupo M, Operschall E, et al. Economic burden of non-cystic fibrosis bronchiectasis in the first year after diagnosis from a US health plan perspective. Appl Health Econ Health Policy. 2013;11:299-304.

28. Gale NS, Bolton CE, Duckers JM, et al. Systemic comorbidities in bronchiectasis. Chron Respir Dis. 2012;9:231-238.

29. Devaraj A, Wells AU, Meister MG, et al. Pulmonary hypertension in patients with bronchiectasis: prognostic significance of CT signs. AJR Am J Roentgenol. 2011;196:13001304.

30. Faria Ju nior NS, Urbano JJ, Santos IR, Silva AS, Perez EA, Souza $\mathrm{A}^{\wedge} \mathrm{H}$, et al. Evaluation of 
obstructive sleep apnea in non-cystic fibrosis bronchiectasis: A cross-sectional study. PLoS ONE.2017;12(10): e0185413.

31. Rusu A, Nita C, Todea D, Rosca L, Bala C, Hancu N. Correlation of the daytime sleepiness with respiratory sleep parameters in patients with sleep apnea and type 2 diabetes. Acta Endocrinologica. 2011;VII(2):163-171.

32. Martinez-Garcia MA, de Gracia J, Vendrell Relat $\mathrm{M}$, et al. Multidimensional approach to noncystic fibrosis bronchiectasis: the FACED score. Eur Respir J.2014;43:1357-1367.

33. Finklea JD, Khan g, Thomas S et al. Predictors of mortality in hospitalized patients with acute exacerbation of bronchiectasis. Respir Med.2010;104:816-21.

34. Chang CC, Singleton RJ, Morris PS et al. Pneumococcal vaccines for children and adults with bronchiectasis. Cochrane Database Syst Rev. 2009; 2:CD006316.

35. Todea D, Cadar O, Simedru D, Roman C, Tanaselia C, Suatean I, Naghiu A, Determination of Major-to-Trace Minerals and Polyphenols in Different Apple Cultivars. Not Bot Horti Agrobo. 2014;42(2):523-529.

36. Dobrosielski DA, Papandreou C, Patil SP, SalasSalvadó J. Diet and exercise in the management of obstructive sleep apnoea and cardiovascular disease risk. Eur Respir Rev.2017;144(26). http://err.ersjournals.com/content/26/144/16011 0.long, Accesed on 1st September 2018.

37. Radescu OD, Albu S, Baciut MS, Coman AC, Bechir ES, Pacurar M, Todea DA. Results in the Treatment with Twin Block Polymeric Appliance of the Retrognathic Mandible in Sleep Apnea Patients. Materiale Plastice.2017;54(3):473-476.

38. Rusu A, Todea D, Rosca L, Nita C, Bala C. The development of a sleep apnea screening program in Romania type 2 diabetic patients: a pilot study. Acta Diabetologica.2012.49(2):105-9.

39. Lee AL, Burge AT, Holland AE. Positive expiratory pressure therapy versus other airway clearance techniques for bronchiectasis. Cochrane Database Syst. Rev. 2017; 9: CD011699.

40. Polverino E, Goeminne PC, McDonnell MJ, Aliberti S, Marshall SE, Loebinger MR, Murris $\mathrm{M}$, Cantón R, Torres A, Dimakou $\mathrm{K}$ et al. European Respiratory Society guidelines for the management of adult bronchiectasis. Eur. Respir. J. 2017; 50: pii: 1700629.

41. Hill AT, Sullivan AL, Chalmers JD, De Soyza A, Elborn JS, Floto AR et al. The BTS Guideline for Bronchiectasis in Adults. Thorax 2019; 74:1 -69.

42. Flude LJ, Aggent P, Bilton D, Chest Physiotherapy techniques in bronchiectasis. Clin Chest Med .2012;33:351-361.

43. Herrero-Cortina B, Aliberti S, Francesco B, Montserrat V, Elborn S, Loebinger M, Menedez $\mathrm{R}$, Torres A, Ringshausen F, Welte T et al. Chest physiotherapy in European patients with bronchiectasis: data from EMBARC registry. Abstract Book - 2nd World Bronchiectasis Conference, Milan, 2017; D23 (100):57.

44. Kim CS, Iglesias AJ, Sackner MA. Mucus clearance by two-phase gas-liquid flow mechanism: asymmetric periodic flow model. J. Appl. Physiol. (1985)1987; 62: 959-71.

45. Andersen JB, Qvist J, Kann T. Recruiting collapsed lung through collateral channels with positive endexpiratory pressure. Scand J Respir Dis. 1979;60: 260-6.

46. Groth S, Stavanger G, Dirksen H, et al. Positive expiratory pressure (PEP-mask) physiotherapy improves ventilation and reduces volume of trapped gas in cystic fibrosis. Clin Respir Physiol .1985;21:339-43.

47. David A. Autogenic drainage- the German approach. In: Pryor JA, editor. Respiratory care. London: Churchill Livingstone; 1991. p. 65-78.

48. Lapin CD. Airway physiology, autogenic drainage, and active cycle of breathing. Respir Care .2002; 47(7):778-85.

49. Patterson JE, Hewitt O, Kent L, Bradbury I, Elborn JS, Bradley JM. Acapella versus 'usual airway clearance' during acute exacerbation in bronchiectasis: a randomized crossover trial. Chron. Respir. Dis. 2007; 4: 67 -74.

50. Thompson CS, Harrison S, Ashley J, Day K, Smith DL. Randomised crossover study of the Flutter device and the active cycle of breathing technique in non-cystic fibrosis bronchiectasis. Thorax 2002; 57: 446-8.

51. Herrero-Cortina B, Vilaro J, Marti D, Torres A, San MiguelPagola M, Alcaraz V, Polverino E. Short-term effects of three slow expiratory airway clearance techniques in patients with bronchiectasis: a randomised crossover trial. Physiotherapy 2016; 102: 357-64. 
52. Munoz G, de Gracia J, Buxo M, Alvarez A, Vendrell M. Long-term benefits of airway clearance in bronchiectasis: a randomised placebo-controlled trial. Eur. Respir. J. 2018; 51: pii: 1701926.

53. Bott J, Blumenthal S, Buxton $\mathrm{M}$, et al. Physiotherapy management of the medical respiratory patient: the adult spontaneously breathing patient. Thorax 2009; 64(Supp1):i2531.

54. Cecins NM, Jenkins SC, Pengelley J, et al. The active cycle of breathing techniques-to tip or not to tip? Respir Med 1999;93:660-5.

55. Lee AL, Burge AT, Holland AE. Airway clearance techniques for bronchiectasis. Cochrane Database Syst. Rev. 2015; (11): CD008351.

56. McIlwaine M, Bradley J, Elborn JS, Moran F. Personalising airway clearance in chronic lung disease. Eur. Respir. Rev. 2017;26: pii: 160086.

57. Coman AC, Todea DA, Popa E, Radu T, Cadar O, Borzan C. Multilateral characterization of masks and tubes surfaces in contact with respiratory system through ventilation, Journal of Optoelectronics and Advanced Materials. 2015;17(9-10):1563-1571.

58. Chin CJ, George C, Lannigan R, Rotenberg B. Association of CPAP Bacterial Colonization with Chronic Rhinosinusitis. J Clin Sleep Med. 2013;8(9):747-750.

59. Todea DA, Suatean I, Coman AC, Rosca LE. The Effect of Climate Change and Air Pollution on Allergenic Potential of Pollens Notulae Botanicae Horti Agrobotanici.2013; 41(2):64650 .

60. Hasani A, Chapman TH, McCool D et al. Domiciliary humidification improves lung mucociliary clearance in patients with bronchiectasis. Chron Respir Dis. 2008; 5:81-6. doi:10.1177/1479972307087190

61. Balsamo R, Lanata L, Egan CG. Mucoactive drugs. Eur. Respir. Rev. 2010; 19: 127-33.

62. Polverino E, Goeminne PC, McDonnell MJ, Aliberti S, Marshall SE, Loebinger MR, Murris M, Cantón R, Torres A, Dimakou $\mathrm{K}$ et al. European Respiratory Society guidelines for the management of adult bronchiectasis. Eur. Respir. J. 2017; 50: pii: 1700629.

63. Hart A, Sugumar K, Milan SJ, Fowler SJ, Crossingham I. Inhaled hyperosmolar agents for bronchiectasis. Cochrane Database Syst. Rev. 2014; (5): CD002996.

64. Wilkinson M, Sugumar K, Milan SJ, Hart A, Crockett A, Crossingham I. Mucolytics for bronchiectasis. Cochrane Database Syst. Rev. 2014; (5): CD001289.

65. Wills P, Greenstone M. Inhaled hyperosmolar agents for bronchiectasis. Cochrane Database Syst. Rev. 2014; (1): CD002996.

66. Olivieri D, Ciaccia A, Marangio E, Marsico S, Todisco T, Del Vita M. Role of bromhexine in exacerbations of bronchiectasis. Double-blind randomized multicenter study versus placebo. Respiration. 1991; 58:117-21.

67. Kellett F, Redfern J, Niven RM. Evaluation of nebulised hypertonic saline (7\%) as an adjunct to physiotherapy in patients with stable bronchiectasis. Respir Med .2005; 99:27-31. doi:10.1016/j. Rmed.2004.05.006.

68. Daviskas E, Anderson SD, gomes K et al. Inhaled mannitol for the treatment of mucociliary dysfunction in patients with bronchiectasis: effect on lung function, health status and sputum. Respirology. 2005;10:46-56.

69. Daviskas E, Anderson SD. Hyperosmolar agents and clearance of mucus in the diseased airway. $\mathrm{J}$. Aerosol Med. 2006; 19:100-9.

70. ISRCTN Register. A $2 \times 2$ factorial randomised open label trial to determine the CLinical and cost-Effectiveness of hypertonic saline (HTS $6 \%$ ) and carbocisteine for Airway cleaRance versus usual care over 52 weeks in bronchiectasis. 2018. [Accessed 11 January 2019] Available online at: https://doi.org/10.1186/ISRCTN89040295

71. Australian New Zealand Clinical Trials Registry. ACTRN12611001199909. The effect of azithromycin and hypertonic saline on quality of life, lung function and exercise capacity in adults with non-cystic fibrosis bronchiectasis. 2012. [Accessed 11 January 2019.] Available online at: https://anzctr.org.au/Trial/Registration/TrialReview. aspx id $=347657$ \&isReview $=$ true

72. Poole P, Black PN, Cates CJ. Mucolytic agents for chronic bronchitis or chronic obstructive pulmonary disease. Cochrane Database Syst. Rev. 2012; (8): CD001287.

73. O'Donnell AE, Barker AF, Ilowite JS et al. Treatment of idiopathic bronchiectasis with aerosolized recombinant human DNase I. 
rhDNase Study group. Chest .1998; 113:132934.

74. Alexescu TG, Maierean A, Ciumarnean L, Budin C, Dogaru G, Todea DA. Rehabilitation therapies in stable chronic obstructive pulmonary disease.2019;10(1):37-44.

75. Pasteur MC, Bilton D, Hill AT, et al. Guideline for non-CF bronchiectasis. Thorax. 2010;65 (Suppl 1):1-58.

76. Feldman C: Bronchiectasis: new approaches to diagnosis and management. Clin Chest Med .2011; 32: 535-546.

77. Anwar GA, Bourke SC, Afolabi G, et al. Effects of long-term low-dose azithromycin in patients with non-CF bronchiectasis. Respir Med .2008;102:1494e96.

78. Evans DJ, Bara AI, Greenstone M. Prolonged antibiotics for purulent bronchiectasis in children and adults. Cochrane Database Syst Rev. 2007;(2):CD001392.

79. Spruit MA, Singh SJ, Garvey C, et al. An official American Thoracic Society/European Respiratory Society statement: key concepts and advances in pulmonary rehabilitation. Am J Respir Crit Care Med .2013;188:13-64.

80. Vremaroiu-Coman A, Alexescu TG, Negrean V, Milaciu MV, Buzoianu AD, Ciumarnean L, Todea DA. Ethical aspects of smoking cessation among the population from Transylvania. Balneo Research Journal.2018;9(3):254-9.

81. Budin CE, Marginean C, Bordea IR, Enache LS, Enache EL, Grigorescu BL, Biro L, Rusu E, Nemes RM, Todea DA. The Influence of Smoking on Nicotine Exposure Biomarkers and Inflammatory Profile Among Foster Care Teenagers, Romania. Rev Chim. 2018;69(12):3659-63.

82. Todea D, Cosma C, Dicu T, Roşca L, Cucoş(Dinu) A, Rişteiu M, Iancu D, Papuc I, Rădulescu D. Lung cancer risk induced by residential radon in CLUJ and Alba Counties, ROMANIA. Environmental Engineering and Management Journal. 2013.12(6):1281-5.
83. Lee AL, Hill CJ, McDonald CF, Holland AE. Pulmonary rehabilitation in individuals with non-cystic fibrosis bronchiectasis: a systematic review. Arch. Phys. Med. Rehabil. 2017; 98: 774-82.

84. Ong HK, Lee All, Hill CJ, Holland AE, Denehy L, Effects of pulmonary rehabilitation in bronchiectasis: A retrospective study, Chronic Respiratory Disease.2011;8(1):21-30.

85. Newall C, Stockley RA, Hill SL. Exercise training and inspiratory muscle training in patients with bronchiectasis. Thorax. 2005;60(11):943-8.

86. Mandal P, Sidhu MK, Kope L, Pollock W, Stevenson LM, Pentland JL, Turnbull K, Mac Quarrie S, Hill AT: A pilot study of pulmonary rehabilitation and chest physiotherapy versus chest physiotherapy alone in bronchiectasis. Respir Med. 2012; 106:1647-1654.

87. Burtin C, Hebestreit H. Rehabilitation in patients with chronic respiratory disease other than chronic obstructive pulmonary disease: exercise and physical activity interventions in cystic fibrosis and non-cystic fibrosis bronchiectasis. Respiration. 2015; 89:181-9.

88. Van Zeller M, Mota PC, Amorim A, Viana P, Martins P, Gaspar L, Hespanhol V, Gomes I. Pulmonary rehabilitation in patients with bronchiectasis: pulmonary function, arterial blood gases, and the 6-minute walk test. J. Cardiopulm. Rehabil. Prev. 2012; 32: 278-83.

89. Lee AL, Hill CJ, Cecins N, Jenkins S, McDonald CF, Burge AT, Rautela L, Stirling RG, Thompson PJ, Holland AE. The short and long term effects of exercise training in non-cystic fibrosis bronchiectasis $-\mathrm{a}$ randomised controlled trial.Respir. Res. 2014; 15: 4.

90. Vivodtzev I, Debigaré R, Gagnon P, Mainguy V, Saey D, Dubé A, Paré ME, Bélanger M, Maltais F. Functional and muscular effects of neuromuscular electrical stimulation in patients with severe COPD: a randomized clinical trial. Chest. 2012;141:716-725. 